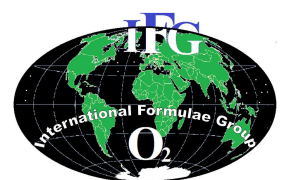

Available online at http://www.ifgdg.org

Int. J. Biol. Chem. Sci. 10(3): 1350-1369, June 2016

International Joumal

of Biological and

Chemical Sciences

ISSN 1997-342X (Online), ISSN 1991-8631 (Print)

Original Paper http://ajol.info/index.php/ijbcs http://indexmedicus.afro.who.int

\title{
Caractéristiques techniques et importance socio-économique de l'apiculture au Nord-Ouest du Bénin : cas de la commune de Cobly
}

\author{
Sfich T. B. AHOUANDJINOU *, Hounnankpon YEDOMONHAN, \\ Aristide C. ADOMOU, Monique G. TOSSOU et Akpovi AKOEGNINOU \\ Laboratoire de Botanique et Ecologie Végétale, Département de Biologie Végétale, Faculté des Sciences et \\ Techniques, Université d'Abomey-Calavi (UAC), 01 BP 4521 Cotonou, Benin. \\ *Auteur correspondant, E-mail : thibaut.ahouandjinou@gmail.com, Tel : (00229) 95634869/62027065
}

\section{REMERCIEMENTS}

Nous remercions sincèrement le gouvernement béninois pour son soutien financier à travers le Programme Appui à la Formation Doctorale du Ministère de l'Enseignement Supérieur et de la Recherche Scientifique.

\section{RESUME}

Au Bénin, la production du miel constitue une source potentielle non négligeable de revenu monétaire pour la population rurale. Une enquête a été conduite au Nord-Ouest du Bénin auprès de 35 apiculteurs pour évaluer les caractéristiques techniques et l'importance socio-économique de l'apiculture. Les apiculteurs enquêtés ont un âge compris entre 20 et 79 ans. La plupart des apiculteurs interviewés $(74,29 \%)$ pratiquaient la chasse au miel avant d'être formés pour l'apiculture moderne. Les types de ruches connus sont la ruche kenyane qui est utilisée exclusivement par $68,57 \%$ des apiculteurs et la ruche traditionnelle utilisée seulement par $8,57 \%$. Le nombre de ruches colonisées par apiculteur ou groupement varie de 3 à 46 . La production annuelle de miel est en moyenne de 10,55 \pm 3,56 litres par ruche et de 148,57 \pm 77,01 litres par apiculteur ou groupement. Le prix de vente du miel est compris entre 1200 et 2000 F CFA par litre. La recette annuelle brute par apiculteur ou groupement varie de 9000 à 580000 F CFA. Le miel est utilisé dans le traitement de 28 maladies dont la brûlure et la toux sont les plus citées.

(C) 2016 International Formulae Group. All rights reserved.

Mots clés: Miel, techniques apicoles, revenu monétaire, usages, Bénin.

\section{Technical characteristics and socio-economic importance of beekeeping in the Northwest of Benin: case of Cobly district}

\begin{abstract}
In Benin, honey production is a significant potential source of cash income for the rural population. A survey was conducted in the Northwest of Benin on 35 beekeepers to assess the technical characteristics and socio-economic importance of beekeeping. Beekeepers surveyed were aged between 20 and 79 years. Most beekeepers interviewed (74.29\%) hunted honey before being trained in modern beekeeping. Known types of
\end{abstract}


hives are the Kenyan hive that is used exclusively by $68.57 \%$ of beekeepers and the traditional hive used only by $8.57 \%$. The number of hives per beekeeper colonized or group ranges from 3 to 46 . The annual honey production is averaging $10.55 \pm 3.56$ liters per hive and $148.57 \pm 77.01$ liters per beekeeper or group. Honey sales price is between 1200 and 2000 FCFA per liter. The gross annual income by beekeeper or group ranges from 9000 to 580,000 CFA francs. Honey is used in the treatment of 28 diseases out of which burn and cough are the most cited.

() 2016 International Formulae Group. All rights reserved.

Keywords: Honey, beekeeping techniques, monetary income, uses, Benin.

\section{INTRODUCTION}

Dans le monde entier, le miel constitue l'un des plus anciens aliments de l'humanité. Il est sans doute, la première source de sucre naturel à l'origine des premières boissons fermentées (Canini et al., 2005). Elaboré par les abeilles à partir du nectar des fleurs ou de sécrétions issues des parties vivantes des plantes (miellat), il connaît de nos jours une vogue nouvelle après une éclipse momentanée due à l'introduction de sucre de betteraves au XIX $^{\mathrm{e}}$ siècle (Djossou et al., 2013). L'apiculture est l'élevage des abeilles pour la production de miel. C'est une activité séculaire dans les pays de l'Afrique de l'Ouest (Paterson, 2008). D'abord basée sur la récolte de la production naturelle dite apiculture de cueillette, elle est passé à l'utilisation des ruches traditionnelles (troncs d'arbres évidés, paille tissée, pot de terre, ruches en tôle, etc.) puis, par la suite, à l'utilisation des ruches à rayons mobiles et à cadres mobiles (ruches kenyanes, ruches Langstroch) dites apiculture moderne (Yédomonhan et al., 2009). L'apiculture moderne génère de nombreux produits de la ruche (miel, propolis, pollen, couvain, venin d'abeilles et gelée royale) en vue de l'exploitation rationnelle de la diversité biologique (Mensah, 2008). Par l'utilisation et la vente de ces produits, elle contribue à améliorer les conditions d'existence de celui qui s'y consacre. Elle contribue aussi à l'économie rurale environnante, non seulement en pollinisant les cultures, mais encore en stimulant les échanges commerciaux.

Depuis 1972, le Bénin a connu l'apiculture moderne avec des ruches à rayons mobiles par Potiron (Hussein, 2001). Mais, l'apiculture moderne n'a véritablement commencé qu'en 1994 avec le Centre Intégré du Bénin pour l'apiculture tropicale. Ce centre a contribué à la reconstitution des populations d'abeilles gravement affectées par la chasse au miel en organisant des stages de formation à l'endroit des apiculteurs. Malgré l'intervention de plusieurs organismes et institutions porteurs de projets de développement rural particulièrement dans le domaine apicole, la production du miel au Bénin reste toujours une activité très peu développée (Paraïso et al., 2012a). Néanmoins, dans certaines régions très pauvres du Bénin, elle fait partie des activités menées par les populations pour satisfaire leurs besoins aussi bien alimentaires, thérapeutiques, qu'économiques.

La production de miel constitue une source potentielle non négligeable de revenus monétaires pour la population rurale (Yédomonhan et al., 2009). Le miel étant l'un des plus importants produits forestiers non ligneux (PFNL) du Bénin, la récente modernisation de la filière du miel a permis d'augmenter la production nationale qui est passée de 4,5 tonnes par an à 23 tonnes par an (FAO, 2012). L'optimisation de la production de miel dans une zone passe par la maîtrise des potentialités techniques de la zone, des 
facteurs climatiques au préalable, de l'importance de l'insertion de l'apiculture dans le secteur agricole, des circuits commerciaux des produits de la ruche et de l'impact de l'apiculture sur les autres activités économiques (Paterson, 2008). En effet, au Nord-Ouest du Bénin, des études ont été réalisées sur les déterminants et les contraintes de la production de miel, puis les perceptions et adaptations des apiculteurs et chasseurs de miel aux changements climatiques (Paraïso et al., 2012a ; 2012b). Par ailleurs, Yédomonhan (2009) a évalué l'importance socioéconomique de la production du miel dans la Lama (zone guinéenne) et à Manigri (zone soudano-guinéenne). Ainsi pour réussir la promotion de la filière apicole au Bénin, il est indispensable d'étendre l'étude de l'importance socio-économique de la production du miel en zone soudanienne.

L'objectif de ce travail est de recenser les pratiques apicoles utilisées et d'évaluer l'importance socio-économique de la production de miel, pour une meilleure diversification des sources de revenus des agro-apiculteurs dans la commune de Cobly.

\section{MATERIEL ET METHODES} Milieu d'étude

L'étude a été conduite dans la commune de Cobly qui est située dans le département de l'Atacora en République du Bénin. D'une superficie de $825 \mathrm{~km}^{2}$, elle est comprise entre les parallèles $0^{\circ} 45^{\prime} 37.2^{\prime}$ ' et $1^{\circ} 04^{\prime} 56.5^{\prime}$ ' de latitude Nord et les méridiens $10^{\circ} 18^{\prime} 25.7^{\prime \prime}$ ' et $10^{\circ} 39^{\prime} 59.7^{\prime}$ ' de longitude Est (Figure 1).

Le climat est de type soudanien à deux saisons dont une saison des pluies de mai à octobre et une saison sèche de novembre à avril (Akoègninou et al., 2006). La pluviosité moyenne annuelle enregistrée au cours de la période de 1980 et 2010 est de $1100 \mathrm{~mm}$. La hauteur maximale des pluies est observée pendant la période de juillet à septembre. La moyenne mensuelle de la température est comprise entre $25,29{ }^{\circ} \mathrm{C}$ en juillet et $32,88^{\circ} \mathrm{C}$ en mars. Les sols sont de types ferrugineux tropicaux (Faure, 1977).

Sur le plan phytogéographique, la commune de Cobly appartient au secteur de l'Atacora occidental de la zone soudanienne où la végétation comprend des savanes boisées, arborées, arbustives et herbeuses ; parsemées de forêts galeries, de lambeaux de forêt dense sèche et de forêt claire, de jachères et de champs (Akoegninou et al., 2006).

Considérant la démographie, la population de Cobly est passée de 46660 habitants en 2002 à 68955 habitants en 2013 soit un taux d'accroissement annuel de 3,53\% (INSAE, 2013). Elle est dominée à $70 \%$ par le groupe ethnique Bèberbè. La principale activité de la population est l'agriculture qui occupe plus de $90 \%$ de la population active. Ensuite viennent, par ordre d'importance l'élevage, le commerce, l'artisanal et l'apiculture (CC, 2004).

\section{Méthode}

Des enquêtes ont été réalisées auprès de 35 apiculteurs de la commune de Cobly (département de l'Atacora). Elles ont été menées par le biais d'un questionnaire semistructuré par des interviews individuelles et en focus group de 5 ou 10 apiculteurs avec l'aide d'un apiculteur interprète. Le focus group permet d'enrichir les informations recueillies par interview individuelle (Lebel et al., 2002). Il est réalisé à l'aide de discussions semi dirigées et fait usage d'une check-list de questions guides flexibles où de nouvelles questions ou pistes d'interrogations émergeant tout au long de l'entretien (Tamboura et al., 1998). Les différentes rubriques du questionnaire sont le profil de l'apiculteur, la connaissance et la technique de la pratique apicole, les atouts et les contraintes financiers 
et environnementaux, les utilités du miel et autres produits de la ruche et les perspectives d'exploitation.

La rentabilité financière de la production de miel a été évaluée à l'aide de la marge nette de la production en utilisant la méthode coûts/recettes (Dieye et al., 2002; Yédomonhan et al., 2009). Les coûts ou dépenses globales de production ont concerné les matériaux et la main d'œuvre; la main d'œuvre familiale étant valorisée au prix du marché. Les recettes brutes ont été estimées à partir des flux monétaires issus de la vente du miel. Les marges nettes ou recettes nettes de la production de miel au terme de la première année d'activité ont été déterminées en déduisant les dépenses globales des recettes brutes.

\section{Analyses statistiques}

Les données collectées ont été codifiées pour une analyse fréquentielle avec le logiciel Microsoft office Excel 2007. Pour un paramètre donné, sa fréquence est le rapport exprimé en pourcentage du nombre d'apiculteurs ayant reconnu ce paramètre par le nombre total d'apiculteurs enquêtés (Yédomonhan et al., 2009). Par ailleurs, la fréquence de citation des recettes à base de miel ou d'identification des plantes mellifères par les apiculteurs est le rapport exprimé en pourcentage du nombre de citations relatives à la maladie ou à l'espèce par le nombre total de citations de toutes les maladies ou des espèces (Dassou et al., 2014).

Afin d'étudier la relation entre le nombre de ruches colonisées et chacune des variables telles que le volume de miel récolté, les dépenses effectuées, les recettes brutes et les marges nettes d'exploitation, une régression linéaire simple a été réalisée. Le test de Student associé à la pente de la régression a été utilisé pour voir la significativité des variations entre les différentes variables étudiées. La normalité des données a été toujours vérifiée par l'analyse des résidus. Le coefficient de corrélation de Pearson (r) est calculé à l'aide du logiciel $R$ version 2.15.3.

\section{RESULTATS}

Caractéristiques structurales et organisationnelles des apiculteurs

L'enquête a montré que l'apiculture est une activité essentiellement exercée par les hommes $(94,29 \%)$. Les femmes ne sont que 2 parmi les enquêtés et représentent $5,71 \%$ des participants à l'enquête. Les apiculteurs enquêtés ont un âge moyen de $40 \pm 11,89$ ans. La tranche d'âge de 40-49 ans présente le plus fort effectif avec $40 \%$ des enquêtés. Ensuite, viennent celles de $30-39$ ans $(28,57 \%)$, de 20 29 ans et de $50-59$ ans $(11,43 \%$ chacune). Les tranches d'âge de 60-69 ans et 70-79 ans renferment respectivement $2,86 \%$ et $5,71 \%$ (Tableau 1).

En considérant l'ethnie, 32 apiculteurs, soit $91,43 \%$ sont des Bèberbès et 3 apiculteurs $(8,57 \%)$ appartiennent à l'ethnie Berba. Sur le plan des catégories socio-professionnelles $65,71 \%$ des personnes enquêtées sont des agriculteurs, suivis des salariés $(25,71 \%)$ et des artisans (8,57\%). Des participants à l'enquête, $31,43 \%$ ne savent ni lire ni écrire. Les restes sont de niveau primaire $(31,43 \%)$, secondaire $(25,71 \%)$ et universitaire $(11,43 \%)$.

Les trois quarts des apiculteurs visités (74,29\%) pratiquaient la chasse au miel avant d'être formés pour l'apiculture moderne. Les autres ont été directement initiés aux techniques modernes, soit par un ami $(14,29 \%)$ ou soit par une ONG $(11,43 \%)$. L'ancienneté des apiculteurs dans l'activité varie de 3 à 24 ans. Plus de la moitié (60\%) a plus de 5 ans d'activité apicole. Les formations et/ou les recyclages ont été donnés 
pour $57,14 \%$ des apiculteurs interviewés par le projet LISA (Lutte Intégrée pour la Sécurité Alimentaire) en 2007 et 2010, suivis de l'ONG BUPDOS (Organisation Non Gouvernementale Bureau des Projets de Développement des Euvres Sociales) pour $31,43 \%$ en 2006 et 2009, le projet CEGEDRAO (Projet de Renforcement des Compétences pour l'Emploi des jeunes et le Développement en Afrique de l'Ouest) pour $14,29 \%$ en 2010 et le projet UNSO (United Nations Sudano-sahelian Organization) pour $5,72 \%$ en 2012. Ces formations leur ont permis de mettre en pratique les nouvelles techniques d'élevage des abeilles. C'est ainsi que $100 \%$ des apiculteurs visités reconnaissent la caste des ouvrières, 94,29\% des enquêtés peuvent distinguer la reine, la seule femelle féconde; $91,43 \%$ des apiculteurs interviewés connaissent la caste des faux bourdons et $62,86 \%$ d'entre eux affirment connaître les couvains (œufs et larves). Les produits récoltables dans la ruche sont aussi diversement connus : $100 \%$ pour le miel, $97,14 \%$ pour la cire et $14,29 \%$ pour la propolis. Les agro-apiculteurs sont très conscients des mauvaises pratiques dont ils ont été les auteurs dans le passé et qui ont des conséquences énormes sur la biodiversité. Ils ont, à 100\%, déclaré être amis et protecteurs des mouches à miel en évoquant comme arguments les biens faits de l'apiculture moderne à savoir : l'augmentation des revenus par la production de miel de bonne qualité, l'amélioration des rendements agricoles, la conservation de la biodiversité et la protection de l'environnement.

La capture des essaims d'abeilles s'effectue en deux (2) périodes différentes de l'année. Pour la plupart des apiculteurs (80\%), elle a lieu en saison sèche (février à avril) et en saison des pluies (septembre à minovembre); pour d'autres (20\%), elle se réalise toute l'année en fonction des conditions climatiques et des perturbations liées à la disponibilité des nutriments et les activités anthropiques dans le milieu.

En ce qui concerne la récolte de miel, $88,57 \%$ des apiculteurs font deux (2) récoltes par an. La première a lieu entre mars et avril de même que, chez les apiculteurs $(11,43 \%)$ qui ne récoltent qu'une seule fois l'an. La seconde récolte s'opère en septembre ou en octobre, lorsque la colonie possède une reine jeune et forte. Les apiculteurs sont organisés en groupement de 2 à 15 personnes.

\section{Technique et pratique apicole utilisées dans la commune de Cobly}

Le Tableau 2 montre différentes variables concernant les pratiques apicoles utilisées par les apiculteurs. Le nombre moyen de ruche colonisée par apiculteurs ou par groupement est de 14,48 \pm 7,49 installés dans un rucher de superficie moyenne de 1,1 \pm 1,56 hectare.

Deux types de ruches sont connus des agro-apiculteurs. La majorité des enquêtés exercent leur activité avec les ruches kenyanes $(68,57 \%)$, ensuite viennent ceux qui possèdent les deux types de ruches (ruches traditionnelles et kenyanes) $(22,86 \%)$ et ceux qui utilisent les ruches traditionnelles $(8,57 \%)$. Les savanes constituent la formation végétale où $37,14 \%$ des apiculteurs installent leurs ruchers. Elles sont suivies des plantations (20\%), des champs $(17,14 \%)$, des forêts $(14$, $29 \%$ ) et des jachères $(11,43 \%)$.

Les appâts utilisés par les apiculteurs sont de gamme variée. Toutefois, la cire reste l'appât le plus employé (94,28\%). Son taux de succès varie de $50 \%$ à $100 \%$ d'un apiculteur à l'autre. La récolte du miel se fait en période de soudure selon leurs habitudes (mars à mimai). Parmi les produits de la ruche, trois seulement sont exploités dans le milieu d'étude. Cent pour cent des apiculteurs récoltent le miel et $97,14 \%$ exploitent la cire. 
La propolis est exploitée par seulement $14,29 \%$ des enquêtés, elle reste de loin le produit le moins recherché dans les ruches. Le volume moyen de miel récolté dans une ruche au cours d'une année est de 10,55 \pm 3,56 litres.

Les activités d'entretien menées régulièrement dans les ruchers consistent à contrôler l'état des ruches, à nettoyer le rucher en fauchant les herbes autour des ruches, à apporter de l'eau au rucher lorsque le rucher est éloigné d'un point d'eau et à réaliser des pare-feux en début de saison sèche.

S'agissant de l'identification des plantes mellifères visitées par les abeilles et servant pour la plupart de lieu de capture des colonies sauvages susceptibles d'être enruchées par les apiculteurs, le Tableau 3 présente la liste des espèces mellifères habituellement rencontrées par les apiculteurs. Au total, 25 espèces mellifères, regroupées en 25 genres et 14 familles ont été recensées. Les familles les plus importantes sont les Leguminosae $(28,57 \%)$, les Anacardiaceae, les Poaceae et les Rubiaceae $(21,42 \%$ chacune). Parkia biglobosa est majoritaire avec un taux de $20,48 \%$ de citation. Elle est suivie des espèces comme Adansonia digitata et Vitellaria paradoxa $(12,65 \%$ chacune), Sarcocephalus latifolius (11,44\%).

\section{Importance économique de la production du miel}

En tenant compte des données statistiques de la production de miel par apiculteur ou groupement (Tableau 4), le nombre de ruches colonisées varie de 3 à 46, avec une moyenne de $14,48 \pm 7,49$. Le prix de confection d'une ruche kenyane est compris entre 15000 et $25000 \mathrm{~F} \mathrm{CFA}$, avec une moyenne $12400 \pm 7405$ F CFA. Le volume de miel récolté est en moyenne $148 \pm$ 77,01 litres par apiculteur ou groupement. En fonction de la période de l'année et de la qualité du miel, le prix de vente du miel fluctue entre 1200 et $2000 \mathrm{~F} \mathrm{CFA}$. Presque la totalité du miel produit $(92,89 \%)$ est destinée à la vente et les recettes brutes qui en découlent, s'élèvent en moyenne à $237037 \pm$ 159167 F CFA/an/apiculteur. Le coût global de la production, relatif à l'équipement durable (ruches, accoutrements, bottes, gants, enfumoirs et plastiques) et rarement à la main d'œuvre est en moyenne de $213371 \pm 189239$ F CFA. Le bénéfice net par producteur est en moyenne de $23665 \pm 132788$ F CFA.

D'après la Figure 2, les facteurs tels que le volume de miel récolté, les dépenses et les recettes brutes ont une relation hautement significative avec le nombre de ruches colonisées $(\mathrm{p}=0,000)$. Le coefficient de corrélation de Pearson (r) est de 0,67 pour le nombre de ruches colonisées et le volume de miel récolté (Figure 2a). L'équation de la droite de régression permettant de prédire le volume de miel (y) à partir du nombre de ruches colonisées $(\mathrm{x})$ est : $\mathrm{y}=6,928 \mathrm{x}+$ 48,208.

Le coefficient de corrélation de Pearson est de 0,70 ( $\mathrm{p}=0,000)$ pour le nombre de ruches colonisées (x) et les dépenses totales effectuées (y) (Figure 2b). L'équation de la droite de régression est $\mathrm{y}=$ $17695 x-42954$.

Le coefficient de corrélation de Pearson est de 0,71 ( $\mathrm{p}=0,000)$ pour le nombre de ruches colonisées (x) et les recettes brutes (y) (Figure 2c). L'équation entre ces deux variables est $\mathrm{y}=15093 \mathrm{x}+18404$.

En considérant que l'ensemble des apiculteurs enquêtés sont à leur première année d'installation et d'exploitation des ruchers, les dépenses totales de la production de miel varient de 0 à $750000 \mathrm{~F}$ CFA par apiculteur. Les recettes brutes générées par la vente de miel s'élèvent de 9000 à $580000 \mathrm{~F}$ CFA et les recettes nettes varient de -302000 F à 245000 F CFA par apiculteur ou 
groupement. Parmi les 24 apiculteurs exploitant uniquement les ruches kenyanes, 5 connaissent des déficits allant de 38000 pour 5 ruches à $302000 \mathrm{~F}$ CFA pour 16 ruches. Les 19 apiculteurs restant réalisent un bénéfice de 15000 F CFA et 245000 F CFA pour respectivement 10 ruches et 21 ruches. $\mathrm{Au}$ même moment, les trois apiculteurs ayant exploité uniquement les ruches traditionnelles effectuent un bénéfice variant de 9000 F CFA pour 3 ruches à $82500 \mathrm{~F} \mathrm{CFA}$ pour 15 ruches. En ce qui concerne les huit apiculteurs possédant les deux types de ruches, un seul connaît un déficit de 120000 F CFA avec une exploitation de 17 ruches, tandis que deux d'entre eux disposant 10 et 13 ruches réalisent respectivement un bénéfice de $15000 \mathrm{~F}$ CFA et 17000 F CFA. Les cinq (5) apiculteurs restants ne connaissent ni de perte et ni de bénéfice. Aucune corrélation linéaire significative $(p>0,05)$ n'existe entre le nombre de ruches colonisées et les marges nettes par apiculteur (Figure 3). La courbe de tendance qui permet de mieux prédire la recette nette, issue de la production de miel (y) en fonction du nombre de ruches colonisées (x), est de type polynomial. L'équation associée à cette courbe est : y = $511,8 x^{2}+20967 x-14471$. Elle ne permet de prédire que $20,5 \%$ de succès de bénéfice en fonction du nombre de ruches colonisées.

Usages alimentaires, thérapeutiques et magiques du miel et autres produits de la ruche dans la commune de Cobly

Le miel est de plus en plus sollicité pour ses multiples utilisations. En alimentation, l'ensemble des apiculteurs interviewés utilisent le miel comme source de sucre pour le café et/ou la bouillie et $11,43 \%$ parmi eux prennent le miel pour conserver les fruits frais $\mathrm{du}$ palmier dattier (Phoenix dactylifera). En médicine traditionnelle, le miel est employé dans le traitement de 28 maladies et symptômes (Tableau 5). La toux et la brûlure sont les affections pour lesquelles le miel est plus utilisé $(16,29 \%$ chacune). Ensuite viennent, par ordre décroissant d'importance, les maux de tête $(10,24 \%$ chacune), la fièvre chez les enfants $(8,43 \%)$, l'insomnie et les maux d'oreilles $(8,02 \%)$, la fatigue intellectuelle $(5,42 \%)$, le paludisme et l'aide mémoire $(4,80 \%$ chacune $)$, l'hypertension $(4,22 \%)$, le rhume et la bonne chance $(2,41 \%)$.

Quant à la forme d'usage, le miel est utilisé à l'état cru dans le traitement de 96,43\% des maladies et symptômes, à l'état pur dans le traitement de $57,43 \%$ des affections. Il est dilué dans d'autres solutions pour $32,14 \%$ et en mélange avec des organes végétaux pour $10,71 \%$ des maladies et symptômes cités.

En ce qui concerne les voies d'administration des remèdes à base de miel, c'est la voie orale qui prédomine avec $82,14 \%$ dont $60,71 \%$ par lapement et $21,43 \%$ comme boisson. On constate que le bain $(7,14 \%)$, le cataplasme et le massage $(3,57 \%$ chacun $)$ représentent la voie externe et l'instillation auriculaire présente une fréquence de 3,57\%.

Pour la posologie, elle varie en fonction de l'affection et de son degré d'évolution. En effet, 57,14\% des maladies doivent être traitées en suivant un rythme de deux à trois prises par jour pendant au moins une semaine de prescription. Le reste $(42,86 \%)$ doit suivre un traitement suivant une prise unique par jour durant une semaine pour un soulagement prompt.

En dehors des utilités alimentaires et médicinales, le miel présente une valeur socio-culturelle pour les populations de la commune de Cobly. Il est associé à d'autres ingrédients au cours des cérémonies de purification citées par $57,14 \%$ des enquêtés pour apaiser les génies en colère, de "Ticonté" qui est un rift de passage de l'adolescence à la vie adulte $(17,14 \%)$. Il est aussi important pour les cérémonies de sortie de nouveau-né selon $5,71 \%$ des enquêtés et d'accueil des 
hôtes de marque dans les familles selon $2,86 \%$ des personnes enquêtées.

\section{Contraintes et perspectives de la production de miel dans la commune de Cobly}

Selon les producteurs enquêtés, les principales contraintes sont par ordre d'importance le manque de ressources financières et les pratiques agricoles $(100 \%$ chacun), l'absence de circuit de commercialisation $(85,71 \%)$, les ennemis des abeilles $(51,41 \%)$ et la destruction des ruches $(22,73 \%)$. Les fourmis et les reptiles $(18,18 \%$ chacun) et les coléoptères $(15,15 \%)$ ont été signalés aussi par les apiculteurs.

L'ensemble des enquêtés (100\%) ont pris l'option de continuer l'apiculture comme activité secondaire avec un nombre moyen de $76,42 \pm 28,42$ ruches et la vulgarisation de l'élevage des abeilles pour la mise en valeur des terres incultes et impropres à l'agriculture. Aussi la totalité des apiculteurs expriment-elles la nécessité de formation de renforcement pour la sélection des colonies produisant plus de miel, l'essaimage artificiel, l'exploitation du pollen et de la gelée royale.

Tableau 1 : Structure des âges des apiculteurs de Cobly.

\begin{tabular}{lcc}
\hline Classe d'âge & Effectif & Pourcentage \\
\hline $20-29$ ans & 4 & 11,43 \\
$30-39$ ans & 10 & 28,57 \\
$40-49$ ans & 14 & 40,00 \\
$50-59$ ans & 4 & 11,43 \\
$60-69$ ans & 1 & 2,86 \\
$70-79$ ans & 2 & 5,71 \\
\hline Total & 35 & 100,00 \\
\hline
\end{tabular}

Tableau 2 : Caractéristiques techniques de l'apiculture dans la commune de Cobly.

\begin{tabular}{llc}
\hline Paramètres & Caractéristiques & Fréquence de citation (\%) \\
\hline \multirow{3}{*}{ Taille de l'exploitation apicole } & Nombre moyen de ruches colonisées & - \\
\hline \multirow{2}{*}{ Aire minimale du rucher } & $14,48 \pm 7,49[3-46$ ruches colonisées $]$ & \\
\hline \multirow{3}{*}{ Type de ruche } & Superficie moyenne & - \\
& $1,1 \pm 1,56$ hectare $[0,25-10$ hectares $]$ & 8,57 \\
& Traditionnel & 68,57 \\
& Kenyane & 22,86 \\
\hline \multirow{4}{*}{ Type de formation végétale } & Mixte & 14,29 \\
abritant les ruchers & Forêt & 37,14 \\
& Savane & \\
& & 11,43 \\
& Jachère & 20 \\
& Plantation & 17,14 \\
\hline & Champ & 94,28 \\
Type d'appât utilisé & Cire & 88,57 \\
& Bouse de vache + cendre & 5,71 \\
& son frais de sorgho (Sorghum bicolor) & 28,57 \\
\hline
\end{tabular}




\begin{tabular}{|c|c|c|}
\hline \multirow[b]{2}{*}{ Récolte du miel } & Mars-avril (période de soudure) & 100 \\
\hline & Septembre-octobre & 71,43 \\
\hline \multirow{3}{*}{ Produits récoltés } & Miel & 100 \\
\hline & Cire & 97,14 \\
\hline & Propolis & 14,29 \\
\hline \multirow{3}{*}{$\begin{array}{l}\text { Activités } \\
\text { ruchers }\end{array}$} & $\begin{array}{l}\text { Contrôle de l'état des ruches et } \\
\text { nettoyage du rucher }\end{array}$ & 74,28 \\
\hline & Apport de l'eau au rucher & 2,86 \\
\hline & Pare-feu & 22,86 \\
\hline Quantité de miel récolté & $\begin{array}{l}\text { Volume moyen de miel par ruche et par an } \\
10,54 \pm 3,50 \text { litres [2-14 litres] }\end{array}$ & - \\
\hline
\end{tabular}

Tableau 3 : Liste des espèces mellifères habituellement rencontrées par les apiculteurs autour de leurs ruchers.

\begin{tabular}{|c|c|c|c|c|}
\hline $\mathbf{N}^{\circ}$ & Espèces végétales & Famille & $\begin{array}{c}\text { Nombre } \\
\text { de citation }\end{array}$ & $\begin{array}{c}\text { Fréquence de } \\
\text { citation }(\%)\end{array}$ \\
\hline & Parkia biglobosa (Jacq.) R.Br. ex & Leguminosae- & & \\
\hline 1 & Benth. & Mimosoideae & 34 & 20,48 \\
\hline 2 & $\begin{array}{l}\text { Adansonia digitata } \mathrm{L} \text {. } \\
\text { Vitellaria naradoxa } \mathrm{C} \text { F Gaertn } \mathrm{ssn}\end{array}$ & Bombacaceae & 21 & 12,65 \\
\hline 3 & $\begin{array}{l}\text { Paradoxa } \\
\text { Sarcocephalus latifolius (Sm.) }\end{array}$ & Sapotaceae & 21 & 12,65 \\
\hline 4 & E.A.Bruce & Rubiaceae & 19 & 11,45 \\
\hline 5 & Gardenia erubescens Stapf \& Hutch. & Rubiaceae & 10 & 6,02 \\
\hline 6 & $\begin{array}{l}\text { Mangifera indica } \mathrm{L} . \\
\text { Senna siamea (Lam.) .S.Irwin \& }\end{array}$ & $\begin{array}{l}\text { Anacardiaceae } \\
\text { Leguminosae- }\end{array}$ & 10 & 6,02 \\
\hline 7 & Barneby & $\begin{array}{l}\text { Caesalpinioideae } \\
\text { Leguminosae- }\end{array}$ & 8 & 4,82 \\
\hline 8 & Acacia sp Mill. & Mimosoideae & 5 & 3,01 \\
\hline 9 & Borassus aethiopum Mart. & $\begin{array}{c}\text { Arecaceae } \\
\text { Leguminosae- }\end{array}$ & 5 & 3,01 \\
\hline 10 & Parkinsonia aculeata $\mathrm{L}$. & Caesalpinioideae & 5 & 3,01 \\
\hline 11 & $\begin{array}{l}\text { Hyphaene thebaica (L.) Mart. } \\
\text { Lannea microcarpa Engl. \& K. }\end{array}$ & Arecaceae & 4 & 2,41 \\
\hline 12 & Krause & Anacardiaceae & 4 & 2,41 \\
\hline 13 & Sorghum bicolor (L.) Moench & Poaceae & 4 & 2,41 \\
\hline 14 & Anacardium occidentale $\mathrm{L}$. & Anacardiaceae & 3 & 1,81 \\
\hline 15 & $\begin{array}{l}\text { Eucalyptus camaldulensis Dehn. } \\
\text { Pennisetum glaucum (L.) R.Br. ssp. }\end{array}$ & Myrtaceae & 2 & 1,20 \\
\hline 16 & glaucum & Poaceae & 2 & 1,20 \\
\hline 17 & Azadirachta indica A.Juss. & Meliaceae & 1 & 0,60 \\
\hline 18 & Balanites aegyptiaca (L.) Delile & Zygophyllaceae & 1 & 0,60 \\
\hline 19 & Cochlospermum planchoni Hook.f. & Cochlospermaceae & 1 & 0,60 \\
\hline 20 & Combretum sp Loefl. & Combretaceae & 1 & 0,60 \\
\hline
\end{tabular}




\begin{tabular}{llccc}
\hline 21 & Gmelina arborea Roxb. & Verbenaceae & 1 & 0,60 \\
22 & Mitracarpus hirtus (L.) De. & Rubiaceae & 1 & 0,60 \\
23 & Moringa oleifera Lam. & Moringaceae & 1 & 0,60 \\
24 & Vitex doniana Sweet & Verbenaceae & 1 & 0,60 \\
25 & Zea mays L. & Poaceae & 1 & 0,60 \\
\hline
\end{tabular}

Tableau 4 : Données statistiques de la production de miel par apiculteur à Cobly.

\begin{tabular}{lc}
\hline Variables & Moyenne \\
\hline Nombre de ruches colonisées & $14,48 \pm 7,49$ \\
Prix de confection d'une ruche & $12400 \pm 7405$ \\
Volume de miel récolté (litre)/an & $148,57 \pm 77,01$ \\
Prix de vente de miel/litre & $1600 \pm 262$ \\
$\begin{array}{l}\text { Coût global de la production au terme } \\
\text { de la première année d'activité }\end{array}$ & $213371 \pm 189239$ \\
$\begin{array}{l}\text { Recette brute au terme de la première } \\
\text { année de production }\end{array}$ & $237037 \pm 159167$ \\
Marges nettes de la production au terme & \\
de la première année d'activité & $23665 \pm 132788$ \\
\hline
\end{tabular}

Tableau 5 : Usages thérapeutiques du miel et des autres produits de la ruche.

\begin{tabular}{lccccc}
\hline $\begin{array}{l}\text { Maladies et } \\
\text { symptômes } \\
\text { traités }\end{array}$ & FU & $\begin{array}{c}\text { Mode de } \\
\text { préparation }\end{array}$ & VA & Posologie & $\begin{array}{c}\text { Fréquence de } \\
\text { citation (\%) }\end{array}$ \\
\hline Toux & Cru & Laper le miel & Orale & $\begin{array}{c}\text { Prendre 1 cuillerée de } \\
\text { miel, matin et soir } \\
\text { jusqu'à la guérison. }\end{array}$ & 16,27 \\
\hline Brûlure & Cru & $\begin{array}{c}\text { Passer le miel sur la } \\
\text { partie brûlée }\end{array}$ & $\begin{array}{c}\text { Externe/ } \\
\text { Cataplasme }\end{array}$ & $\begin{array}{c}\text { Passer au moins 3 fois } \\
\text { par jour pendant 3 jours } \\
\text { successifs. }\end{array}$ & 16,27 \\
\hline Maux de tête & Cru & Laper le miel & Orale & $\begin{array}{c}\text { Laper régulièrement au } \\
\text { moins 1 cuillerée de } \\
\text { miel par jour. }\end{array}$ & 10,24 \\
\hline Fièvre chez les & Cru & $\begin{array}{c}\text { Passer le miel sur le } \\
\text { enfants }\end{array}$ & Externe/ & $\begin{array}{c}\text { Une fois par jour } \\
\text { jusqu'à ce que la } \\
\text { température corporelle } \\
\text { se régularise. }\end{array}$ & 8,43 \\
\hline Insomnie & Cru & $\begin{array}{c}\text { Mélanger 4 cuillerées } \\
\text { de miel + un verre de } \\
\text { lait de vache frais }\end{array}$ & Orale & $\begin{array}{c}\text { Prendre le mélange une } \\
\text { fois par jour durant une } \\
\text { semaine. }\end{array}$ & 6,02 \\
\hline Maux d'oreille & Cru & $\begin{array}{c}\text { Imbiber un morceau de } \\
\text { coton dans du miel }\end{array}$ & Auditive & $\begin{array}{c}\text { Introduire le coton } \\
\text { imbibé de miel dans } \\
\text { l'oreille }\end{array}$ & 6,02 \\
\hline
\end{tabular}




\begin{tabular}{|c|c|c|c|c|c|}
\hline & & & & $\begin{array}{l}\text { le matin et le soir au } \\
\text { coucher pendant une } \\
\text { semaine. }\end{array}$ & \\
\hline $\begin{array}{l}\text { Fatigue } \\
\text { intellectuelle }\end{array}$ & Cru & $\begin{array}{c}\text { Ajouter du miel dans } \\
\text { du jus de citron (Citrus } \\
\text { limon) dilué }\end{array}$ & Orale & $\begin{array}{c}\text { Boire le mélange } \\
\text { obtenu } \\
\text { matin et soir pendant } \\
\text { une } \\
\text { semaine }\end{array}$ & 5,42 \\
\hline Paludisme & Cru & $\begin{array}{c}\text { Ajouter } 2 \text { cuillerées à } \\
\text { soupe de miel aux } \\
\text { tisanes }\end{array}$ & Orale & $\begin{array}{l}\text { Boire } 1 \text { verre matin et } \\
\text { soir } \\
\text { pendant une semaine }\end{array}$ & 4,82 \\
\hline Aide-mémoire & Cru & $\begin{array}{l}\text { Mélanger le miel + la } \\
\text { poudre des pépins de } \\
\text { papaye (Carica } \\
\text { papaya) non mûres }\end{array}$ & Orale & $\begin{array}{c}\text { Laper souvent } 1 \\
\text { cuillerée à } \\
\text { soupe le matin à jeun. }\end{array}$ & 4,79 \\
\hline Hypertension & Cru & $\begin{array}{l}\text { Laisser un morceau de } \\
\text { sucre dans du miel } \\
\text { pendant un jour }\end{array}$ & Orale & $\begin{array}{l}\text { Mettre le morceau de } \\
\text { sucre } \\
\text { dans la bouche et } \\
\text { respirer. }\end{array}$ & 4,22 \\
\hline Bonne chance & Cru & $\begin{array}{l}\text { Mélanger le miel avec } \\
\text { d'autres ingrédients }\end{array}$ & Orale & $\begin{array}{c}\text { Laper le mélange le } \\
\text { matin. }\end{array}$ & 2,41 \\
\hline Rhume & Cru & $\begin{array}{c}\text { Mélanger } 3 \text { cuillerées } \\
\text { de miel }+3 \text { cuillerées } \\
\text { de jus de citron ( } \text { Citrus } \\
\text { limon) }\end{array}$ & Orale & $\begin{array}{l}\text { Boire le mélange } \\
\text { obtenu matin } \\
\text { et soir pendant une } \\
\text { semaine. }\end{array}$ & 2,41 \\
\hline Maux de ventre & Cru & $\begin{array}{l}\text { Ajouter du miel dans } \\
\text { un verre d'eau tiède }\end{array}$ & Orale & $\begin{array}{l}\text { Boire la solution } 3 \text { fois } \\
\text { par jour pendant } 3 \text { jours }\end{array}$ & 1,2 \\
\hline $\begin{array}{l}\text { Faiblesse } \\
\text { physique et/ou } \\
\text { convalescence }\end{array}$ & Cru & $\begin{array}{l}\text { Mélanger un œuf + } 3 \\
\text { cuillerée de miel }\end{array}$ & Orale & $\begin{array}{l}\text { Boire le mélange à jeun } \\
\text { le matin pendant } 7 \text { jours }\end{array}$ & 1,2 \\
\hline $\begin{array}{l}\text { Infections } \\
\text { pulmonaires }\end{array}$ & Cru & $\begin{array}{c}\text { Dissoudre la propolis } \\
\text { dans l'alcool et ajouter } \\
\text { du miel }\end{array}$ & Orale & $\begin{array}{c}\text { Prendre } 2 \text { gouttes } \\
\text { (enfant) ou } 4 \text { gouttes } \\
\text { (adulte) sur la langue et } \\
\text { respirer profondément } \\
\text { une fois par jour } \\
\text { pendant un mois }\end{array}$ & 1,2 \\
\hline Plaies purulentes & Cru & $\begin{array}{l}\text { Ecraser la propolis } \\
\text { pour obtenir une } \\
\text { poudre }\end{array}$ & $\begin{array}{c}\text { Externe/ } \\
\text { Cataplasme }\end{array}$ & $\begin{array}{l}\text { Nettoyer la plaie et } \\
\text { mettre la } \\
\text { poudre dans la plaie }\end{array}$ & 1,2 \\
\hline $\begin{array}{l}\text { Dentition chez } \\
\text { les enfants }\end{array}$ & Cru & $\begin{array}{l}\text { Passer le miel sur les } \\
\text { gencives }\end{array}$ & Orale & $\begin{array}{l}\text { Appliquer du miel sur } \\
\text { les gencives le matin, le } \\
\text { midi et le soir pendant } \\
\text { au moins une semaine }\end{array}$ & 1,2 \\
\hline $\begin{array}{l}\text { Dermatoses chez } \\
\text { les enfants }\end{array}$ & Cuit & $\begin{array}{l}\text { Préparer le rayon de } \\
\text { miel + la tisane de }\end{array}$ & $\begin{array}{l}\text { Externe/ } \\
\text { Bain }\end{array}$ & $\begin{array}{l}\text { Faire la toilette au bébé } \\
\text { avec la décoction matin }\end{array}$ & 0,6 \\
\hline
\end{tabular}




\begin{tabular}{|c|c|c|c|c|c|}
\hline & & toilette & & et soir pendant 3 mois & \\
\hline Maux de cœur & Cru & Laper le miel & Orale & $\begin{array}{l}\text { Prendre } 1 \text { cuillerée à } \\
\text { soupe de miel une fois } \\
\text { par jour et } \\
\text { régulièrement }\end{array}$ & 0,6 \\
\hline Ulcère & Cru & Laper le miel & Orale & $\begin{array}{l}\text { Prendre } 2 \text { cuillerées à } \\
\text { jeun tous les matins } \\
\text { jusqu'au soulagement }\end{array}$ & 0,6 \\
\hline Vieillissement & Cru & Laper le miel & Orale & $\begin{array}{c}\text { Consommer le miel de } \\
\text { temps en temps }\end{array}$ & 0,6 \\
\hline $\begin{array}{l}\text { Règles } \\
\text { douloureuses }\end{array}$ & Cru & Laper le miel & Orale & $\begin{array}{c}\text { Laper } 1 \text { cuillerée de } \\
\text { miel matin, midi et soir } \\
\text { une semaine avant les } \\
\text { menstrues }\end{array}$ & 0,6 \\
\hline Asthme & Cru & $\begin{array}{c}\text { Mélanger } 100 \text { ml du } \\
\text { macéré } \\
\text { d'oignon (Allium cepa) } \\
+1 \text { cuillerée à soupe } \\
\text { de jus de citron (Citrus } \\
\text { limon) + } 1 \text { cuillerée à } \\
\text { soupe de miel/Laper le } \\
\text { miel le matin à jeun }\end{array}$ & Orale & $\begin{array}{l}\text { Prendre le mélange le } \\
\text { matin à jeun/1 cuillerée } \\
\text { par jour pendant } 1 \\
\text { semaine }\end{array}$ & 0,6 \\
\hline Maux de foie & Cru & $\begin{array}{l}\text { Laper le miel une } \\
\text { heure avant le repas }\end{array}$ & Orale & $\begin{array}{l}\text { Prendre régulièrement } 1 \\
\text { cuillerée à soupe matin } \\
\text { et soir }\end{array}$ & 0,6 \\
\hline Frigidité & Cru & Laper le miel & Orale & $\begin{array}{l}\text { Prendre régulièrement } 1 \\
\text { cuillerée à soupe matin } \\
\text { et soir }\end{array}$ & 0,6 \\
\hline Ménopause & Cru & Laper le miel & Orale & $\begin{array}{c}\text { Prendre régulièrement } 1 \\
\text { cuillerée à soupe tous } \\
\text { les matins }\end{array}$ & 0,6 \\
\hline Amaigrissement & Cru & $\begin{array}{l}\text { Mélanger un verre } \\
\text { d'eau tiède }+2 \\
\text { cuillerées à soupe }\end{array}$ & Orale & $\begin{array}{l}\text { Prendre régulièrement } \\
\text { le mélange le matin à } \\
\text { jeun et le soir au } \\
\text { coucher }\end{array}$ & 0,6 \\
\hline Prostate & Cru & Laper le miel & Orale & Pendre souvent le miel & 0,6 \\
\hline
\end{tabular}




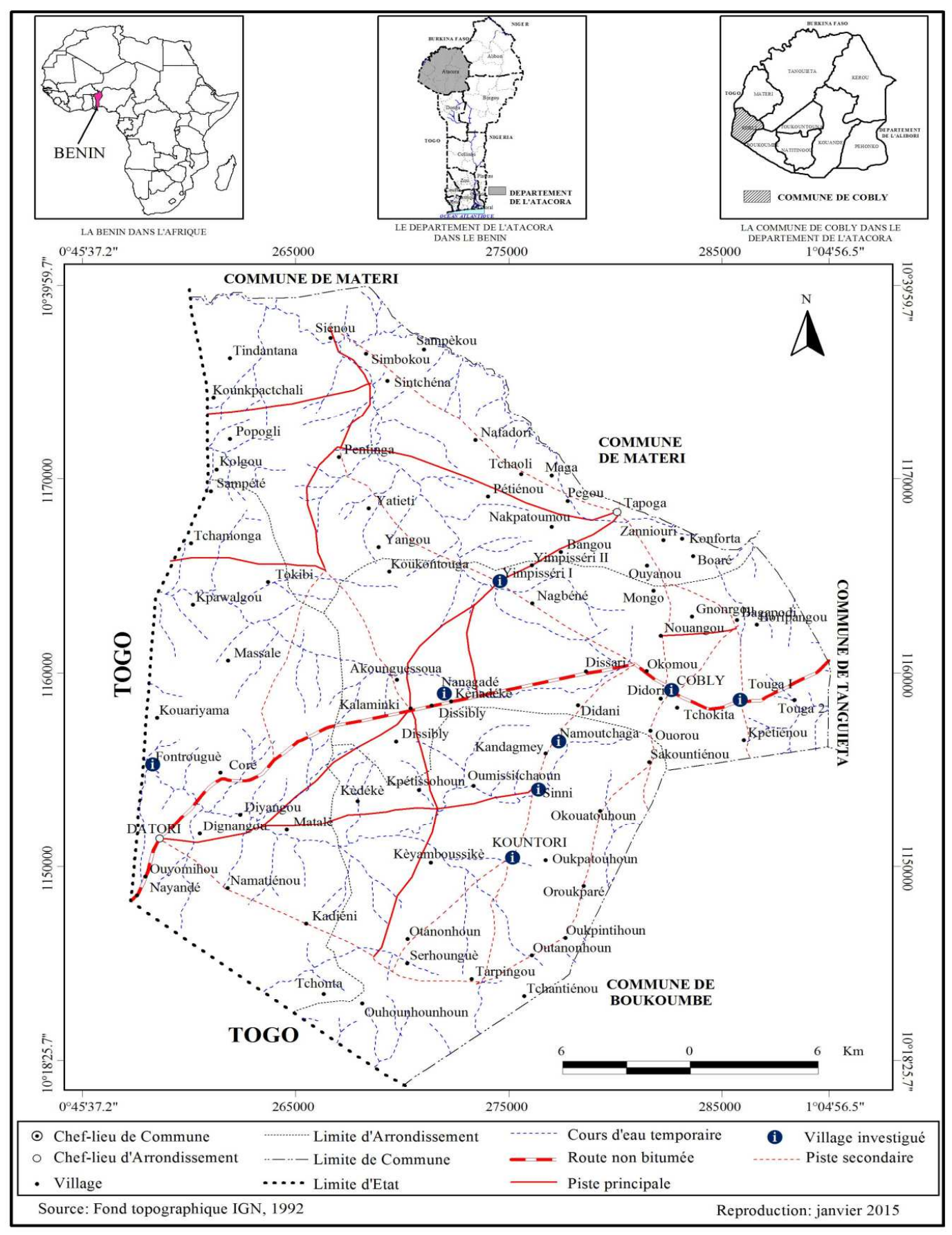

Figure 1 : Localisation de la commune de Cobly au Bénin. 
S. T. B. AHOUANDJINOU et al. / Int. J. Biol. Chem. Sci. 10(3): 1350-1369, 2016

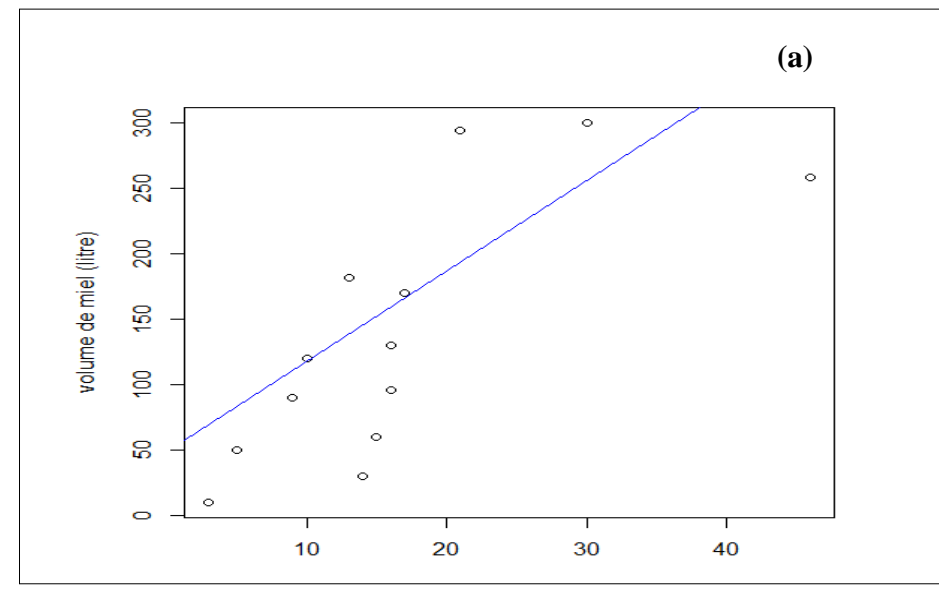

$$
\begin{aligned}
& y=6,928 x+48,208 \\
& R^{2}=45,49 \% \\
& r=0,67 * * * \\
& p<0,001
\end{aligned}
$$

(b)

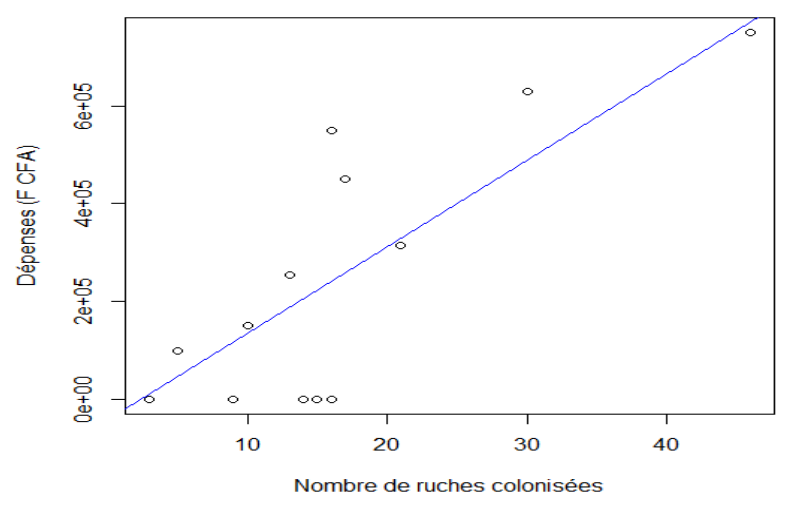

$\mathrm{y}=17695 \mathrm{x}-42954$

$\mathrm{R}^{2}=49,14 \%$

$\mathrm{r}=0,70^{* * * *}$

$\mathrm{p}<0,001$

(c)

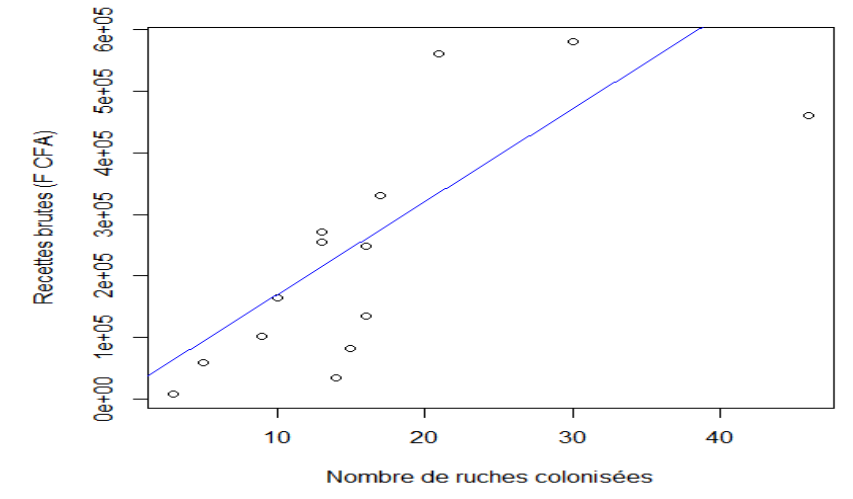

$\mathrm{y}=15093 \mathrm{x}+18404$

$\mathrm{R}^{2}=50,53 \%$

$\mathrm{r}=0,71 * * *$

$\mathrm{p}<0,001$

Figure 2 : Relation entre le nombre de ruches colonisées et diverses variables. a : volume de miel récolté ; $\mathbf{b}$ : dépenses effectuées; $\mathbf{c}$ : recettes brutes; $\mathrm{R}^{2}$ : coefficient de détermination $; \mathrm{r}$ : coefficient de corrélation de Pearson ; p : seuil de probabilité ; $* * *$ : significatif à p $<0,001$. 


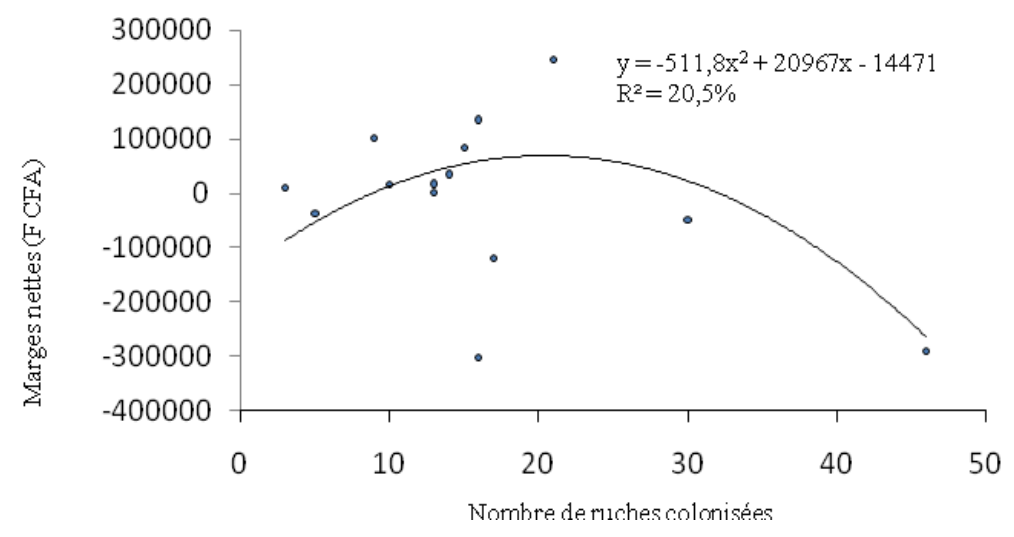

Figure 3 : Corrélation entre le nombre de ruches colonisées et les recettes nettes au terme de la première année d'installation et d'exploitation des ruchers. $\mathrm{R}^{2}$ : coefficient de détermination.

\section{DISCUSSION}

La présente étude a révélé que l'apiculture est pratiquée par les personnes ayant entre 20 et 79 ans à Cobly. Les jeunes scolarisés (élèves et étudiants) de 20 à 29 ans la pratiquent. Ceci est contraire aux constats faits dans la Lama et à Manigri par Yédomonhan (2009) qui indiquaient que les jeunes de moins de 30 ans ne pratiquaient pas l'apiculture. Par ailleurs, la majeure partie de la couche juvénile (agriculteurs et artisans ayant moins de 30 ans) ne la pratique pas. Pourtant elle constitue une proportion relativement élevée $(13,57 \%)$ de la population de la commune de Cobly par rapport à celle des apiculteurs ayant un âge compris entre 30 et 79 ans qui est de 25,70\% (CC, 2006). Ces bras valides préféreraient être utilisés comme mains d'œuvres agricoles à l'intérieur du pays dans les zones cotonnières (Borgou) ou à l'extérieur de nos frontières dans les pays de la sous région comme le Nigeria, le Ghana et le Togo. L'intérêt qu'accordent les jeunes collégiens et bacheliers à l'apiculture constitue un atout important à la formation en apiculture moderne, la promotion et l'intensification de la production de miel à Cobly. Le nombre d'apiculteurs ayant entre 30 et 49 ans est très élevé $(68,57 \%)$. Ceci s'expliquerait par le fait que l'apiculture est considérée comme une activité secondaire à l'agriculture (Olivier, 2008), exercée par cette tranche d'âge qui constitue la grande partie de la population active et ayant de lourdes responsabilités envers leur ménage $(\mathrm{CC}$, 2010). Environ 35\% des apiculteurs visités sont des agents de l'Etat et des artisans. Ceci représente un atout au développement de l'apiculture moderne car l'optimisation de rendement exige que le producteur dispose d'un nombre suffisant de ruches colonisées (Yédomonhan et al., 2009). Ce qui implique des moyens financiers d'installation qui sont à la portée de cette tranche professionnelle des apiculteurs de Cobly.

La plupart des apiculteurs (60\%) a cinq années d'activité apicole. Ceci montre que l'apiculture moderne est une activité récente dans la commune de Cobly comme l'a signalé Hussein (2001). Ainsi, les apiculteurs ont acquis les connaissances théoriques et techniques modernes de l'apiculture par les formations dispensées par les projets LISA (Lutte Intégrée pour la Sécurité Alimentaire), CEGEDRAO (Projet de Renforcement des Compétences pour l'Emploi des jeunes et le Développement en Afrique de l'Ouest) et UNSO (United Nations Sudano-sahelian Organization) et l'ONG BUPDOS (Organisation Non Gouvernementale Bureau 
des Projets de Développement des Euvres Sociales) entre 2006 et 2012. Ces structures ont pour objectif de promouvoir des activités complémentaires aux travaux agricoles dans les milieux ruraux comme l'apiculture moderne, afin de renforcer les capacités des agriculteurs, de lutter contre l'insécurité alimentaire et de préserver les ressources naturelles comme les plantes mellifères, socle d'une assurance de récolte de quantité optimale de miel (Dongock et al., 2008). Mieux, 62,86\% des apiculteurs interviewés font partie des groupements à caractères socio-économique importante pour la production du miel (Yédomonhan et al., 2009).

Le nombre moyen de 14 ruches montre que les exploitations apicoles de Cobly sont de petite taille comme celles de la Lama (7 ruches) et de Manigri (11 ruches) respectivement au sud et au centre Bénin (Yédomonhan, 2009) comparativement à celles en Centrafrique où la moyenne est de 40 à 70 ruches par apiculteur (MbétidBessane, 2004). De même ce nombre signale que les apiculteurs de Cobly sont des producteurs familiaux tout comme ceux de la France dont le nombre de ruches est compris entre 1 et 30 (Gerster, 2012). Le volume moyen annuel de miel récolté $(148,57 \pm 77,01$ litres) par apiculteur de Cobly est supérieur à ceux de 112,68 litres et de 125 litres respectivement obtenus dans les exploitations apicoles semi modernes au Nord-Ouest du Cameroun et par les producteurs de Manigri (Tsafack et al., 2011; Yédomonhan et al., 2009). Par contre, ce volume reste inférieur à celui de 480 litres pour les petits producteurs centrafricains possédant 40 ruches et de 840 litres pour les gros producteurs avec 70 ruches (Mbétid-Bessane, 2004). Ceci s'expliquerait par le fait que tous les apiculteurs enquêtés considèrent l'apiculture comme une activité secondaire à Cobly (CC, $2004 ; 2010)$. La professionnalisation de ces apiculteurs constitue une nécessité pour accroître la taille des ruchers et mieux maîtriser les facteurs déterminants dans la productivité des ruches pour l'optimisation de leurs revenus monétaires annuels (Jansens et al., 2006).

Le volume moyen de miel récolté par ruche, de 10,55 $\pm 3,56$ litres indique un bon rendement dans le milieu d'étude. Ce rendement moyen en miel par ruche est similaire à celui de Manigri qui est de 11,2 \pm 3,7 litres (Yédomonhan et al., 2009) et des hautes terres de l'Ouest du Cameroun qui est de 10 litres (Tchoumboue et al., 2001). Mais il est nettement supérieur à celui de 3,95 litres obtenu dans la Lama (Yédomonhan, 2009) et à celui de 8,4 à $15 \mathrm{~kg}$ (soit environ 4 à 7,5 litres) de miel indiqué pour l'Afrique de l'Ouest (Hussein, 2001). Il révèle une disponibilité des colonies sauvages de l'abeille locale susceptibles d'être enruchées par des apiculteurs dans des ruches modernes et/ou traditionnelles, une disponibilité en plantes mellifères et en nutriments tout le long de l'année permettant une bonne activité continue de butinage des abeilles dans la zone de Cobly (Nombré, 2003). Ceci confirme que les potentialités de production de miel d'une région sont déterminées par de nombreux facteurs dont le type de ruche, la race d'abeilles, l'importance des formations naturelles plurispécifiques constituées d'abondantes ressources nutritionnelles pour les abeilles et la maitrise du calendrier floral (Nombré, 2003 ; 2011).

Le miel récolté $(92,89 \%)$ est principalement destiné à la commercialisation pour l'amélioration des revenus monétaires. Les marges nettes annuelles générées par la production du miel, par apiculteur, varient de 302000 à $245000 \mathrm{~F} \mathrm{CFA}$ avec une moyenne de $23665 \pm 132788$ F CFA. Cette moyenne est nettement supérieure à celle obtenue à Manigri qui est de - $60555 \pm 47140 \mathrm{~F} \mathrm{CFA}$ (Yédomonhan et al., 2009). Ceci révèle qu'une proportion relativement importante $(31,42 \%)$ des apiculteurs n'a pas pu réaliser de bénéfice au terme de la première année 
d'exploitation. Ce constat s'expliquerait par le coût relativement élevé de l'acquisition et de l'installation du matériel que les recettes brutes n'ont pas couvert au cours de la première année de production. Par ailleurs, les agro-apiculteurs ayant réalisé un bénéfice $(68,57 \%)$, sont ceux qui ont reçu des kits apicoles en don et ceux d'un groupement d'apiculteurs constitué des artisans (menuisiers, maçons) et de salariés qui confectionnent leur ruche à partir de leur savoir faire. Ceci montre que l'accroissement du nombre de ruche et la rentabilité de l'apiculture moderne se trouvent à la portée de ces couches socioprofessionnelles, lorsqu'elles se mettent en groupement. Les dépenses totales essentiellement consacrées à l'acquisition de l'équipement durable varient de 98000 à 750000 F CFA pour respectivement 7 et 50 ruches kenyanes. A l'exclusion de don de kit apicole aux agroapiculteurs, et avec le revenu annuel national en milieu rural compris entre 74055 et 125491 F CFA (MAEP, 2001), l'autofinancement en apiculture n'est donc pas à la portée des agroapiculteurs de miel. Ainsi, le développement de l'apiculture implique la prise en charge du financement du matériel d'installation par les structures de développement rural accompagné de suivi, d'assistance et d'évaluation en gestion financière pour leur inculquer les bases de la gestion financière.

La fréquence relativement élevée $(16,27 \%)$ de citation reconnue par les apiculteurs interviewés du traitement de la toux et de la brûlure par le miel confirme les résultats obtenus par Donou Hounsodé (2007) qui révèlent que les brûlures $(100 \%)$ et la toux $(93,3 \%)$ sont les affections les plus courantes dans le département de l'Atacora dont le miel est sollicité pour leur traitement. De même, la toux est la maladie ayant une fréquence élevée de reconnaissance par les apiculteurs dans la Lama $(46,70 \%)$ et à Manigri $(88,57 \%)$ (Yédomonhan, 2009). Tout ceci se justifie par l'efficacité du traitement à base de miel à l'encontre de ces affections. Ce constat s'expliquerait par les nombreuses propriétés antibactérienne et anti-inflammatoire du miel, et son effet osmotique qui diminuent la douleur et l'inflammation puis favorisent la formation de tissus de granulation et ainsi la cicatrisation rapide de la plaie (Hoyet, 2005). De plus, le miel contient des antibiotiques appelés généralement sous le nom générique d'inhibine. En plus du peroxyde d'hydrogène, principale inhibine contenue dans le miel, il existe des inhibines non peroxydes telles que les acides, les bases et les substances neutres et volatiles (Molan, 2001; Bogdanov et al., 2001) qui proviennent en partie des plantes et qui sont de puissants bactériostatiques. Par ailleurs, le miel possède des effets antitussifs, expectorants et adoucissants qui aident à lutter contre les affections respiratoires (Bogdanov, 2015). Egalement, les plantes visitées par les abeilles contiennent des huiles essentielles qui sont bien connues pour leurs effets antiseptiques de la sphère respiratoire (Hoyet, 2005). En ce qui concerne l'apithérapie et plus précisément les recettes issues des enquêtes ethnoapicoles au Bénin (Donou Hounsodé, 2007; Yédomonhan, 2009), 14 vertus thérapeutiques sont reconnues par la présente étude. Les 14 restant constituent l'apport de la présente étude dans la connaissance des usages médico-magiques du miel au Bénin. Ceci pourrait être lié au fait que dans le milieu d'étude, on note une prédominance du groupe socioculturel des Berberbès $(70 \%)$ qu'on ne retrouve que dans cette partie Nord-Ouest du Bénin (CC, 2006).

Les principales contraintes sont d'ordre économique (cherté d'équipement et manque de marché d'écoulement), sociologique (vol de miel) et biologique (ennemis d'abeilles) comme il a été signalé par Paraïso et al. (2012a) dans les communes de Tanguiéta et de Natitingou au Nord-Ouest du Bénin. De même ces contraintes ont été évoquées par les apiculteurs des hautes terres de l'Ouest du Cameroun (Tchoumboue et al., 
2001). Ceci implique qu'elles constituent un frein au développement de l'apiculture moderne dans la zone d'étude.

\section{Conclusion}

La production de miel à Cobly est basée sur les techniques traditionnelles et modernes de l'apiculture. L'objectif principal de l'apiculture reste la production et la vente du miel. Ceci implique que l'élevage des abeilles contribue à l'amélioration des conditions d'existence des populations rurales. Bien que l'apiculture moderne connaisse un regain d'intérêt de la part des agro-apiculteurs et des structures de développement local, le manque de financement et l'absence de circuit de commercialisation constituent un frein à l'accroissement de la production de miel. Il ressort de cette étude que le décollage de l'apiculture moderne nécessite une attention particulière de la part des autorités gouvernementales pour une organisation convenable de la filière apicole, gage d'un développement durable et de la protection du patrimoine naturel.

\section{CONFLIT D'INTERET}

Les auteurs de ce manuscrit déclarent qu'il n'y a aucun conflit d'intérêt.

\section{CONTRIBUTIONS DES AUTEURS}

STBA a assuré la conception de la méthodologie, la collecte et le traitement des données puis la rédaction du manuscrit. Les autres co-auteurs ont contribué à la relecture de la méthodologie et des différentes versions du manuscrit pour l'amélioration de sa qualité scientifique.

\section{REMERCIEMENTS}

Les auteurs remercient sincèrement les apiculteurs de la commune de Cobly pour leur disponibilité et leur franche collaboration au cours de la collecte des données de terrain.

\section{REFERENCES}

Akoègninou $\mathrm{A}$, van der Burg $\mathrm{WJ}$, van der Maesen LJG. 2006. Flore Analytique du Bénin. Backhuys Publishers: Wageningen.

Bogdanov S, Blumer P. 2001. Propriétés antibiotiques naturelles du miel. RSA., 98(3): 107-114. Disponible en ligne sur https://www.google.com/search?q=Bogd anov+S+et+Blumer+P.+2001.pdf

Bogdanov S. 2015. Honey in Medicine. Bee Product Science, p. 25. Disponible en ligne sur http://www.bee-hexagon.net

Canini A, De Santis L, Leonardi D, Di Giustino P, Abbale F, Damesse E, Cozzani R. 2005. Qualificazione dei mielie e piante nettarifere del Camerun Occidentale. La Rivista di Scienza dell'Alimentazione, anno 34n, 4.

CC (Commune de Cobly). 2004. Etude sur le rôle des communes dans la promotion économique et la valorisation des filières porteuse. Monographie de la commune de Cobly : Cobly, p. 61.

CC (Commune de Cobly). 2006. Plan de Développement de la Commune de Cobly ( $1^{\text {ère }}$ génération). Mairie de la commune de Cobly : Cobly ; 96.

CC (Commune de Cobly). 2010. Plan de Développement de la Commune de Cobly ( $2^{\mathrm{e}}$ génération). Mairie de la commune de Cobly: Cobly ; 201.

Dassou HG, Ogni CA, Yédomonhan H, Adomou AC, Tossou GM, Dougnon JT, Akoègninou A. 2014. Diversité, usages vétérinaires et vulnérabilité des plantes médicinales au Nord-Bénin. Int. J. Biol. Chem. Sci., 8(1): 189-210. DOI : http://dx.doi.org/10.4314/ijbcs.v8i1.18

Dieye PN, Faye A, Seydi M, Cissé SA. 2002. Production laitière périurbaine et amélioration des revenus des petits producteurs en milieu rural au Sénégal. Cahiers Agricultures, 11: 251-257. Disponible en ligne sur 
http://www.jle.com/fr/revues/agr/somma ire.phtml?cle_parution=753

Djossou JA, Tchobo FP, Yédomonhan H, Alitonou AG, Soumanou MM. 2013. Evaluation des caractéristiques physicochimiques des miels commercialisés à Cotonou. Tropicultura., 31(3): 163-169. Disponible en ligne sur http://www.tropicultura.org/text/v31n3/1 63.pdf

Dongock DN, Tchoumboue J, Pinta JY, Zango P. 2008. Caractéristiques polliniques des plantes mellifères de la zone soudano-guinéenne d'altitude de l'Ouest Cameroun. Tropicultura, 26(3): 150-154. Disponible en ligne sur http://www.tropicultura.org/content/v26 n3.html.pdf

Donou Hounsodé TM. 2007. Inventaire des Arthropodes ennemis naturels des abeilles, et déprédateurs des produits de la ruche dans les exploitations apicoles du département de l'Atacora au NordOuest du Bénin. Thèse d'ingénieur agronome, Université d'Abomey-Calavi, Abomey-Calavi, p. 149.

FAO. 2012. Produits forestiers non ligneux. Rapport provisoire PFNL, p. 14. Disponible en ligne sur http://www.fao.org/forestry/nwfp/78836/ fr/ben/.pdf

Faure P. 1977. Carte pédologique de reconnaissance de la République Populaire du Bénin à1/200 000 : Feuille de Natitingou et de Porga. ORSTOM., notice explicative, 66(6 et 8): p. 68. Disponible en ligne sur http://www.horizon.documentation.ird.fr lexl-

doc/pleins_textes/pleins...5/notexp/0873 6.pdf

Gerster F. 2012. Plan de développement durable de l'apiculture. Conseil général de l'alimentation, de l'agriculture et des espaces ruraux CGAAER $N^{\circ} 11174-0$, Paris, p. 31. Disponible en ligne sur http://www.agriculture.gouv.fr/ministere /rapport-sur-le-developpement-durablede-lapiculture

Hoyet C. 2005. Le miel : de la source à la thérapeutique. Thèse d'Etat, Université Henri Poincaré-Nancy 1, Nancy, p. 96.

Hussein MH. 2001. L'apiculture en Afrique. Apiacta., 1: 34-48. Disponible en ligne sur http://www.apimondiafoundation. org/pdf

INSAE. 2013. Recensement Général de la Population et de l'Habitat (Résultats Provisoires). MDAEP. INSAE: Cotonou, Bénin ; 8.

Janssens X, Bruneau E, Lebrun P. 2006. Prévision des potentialités de production de miel à l'échelle d'un rucher au moyen d'un système d'information géographique. Apidologie., 37: 351-365. Disponible en ligne sur https://www.hal.archives-ouvertes.fr/hal00892190/document

Lebel F, Debailleul G, Samba SAN, Olivier A. 2002. Contribution des produits forestiers non- ligneux à l'économie des ménages de la région de Thiès, au Sénégal. In Acte 2ème Atelier Régional sur les Aspects socio-économiques de l'agroforesterie au Sahel. Bamako ; 1-9.

MAEP. 2001. Etude sur les Conditions de Vie des Ménages Ruraux: Profil de Pauvreté Rurale et Caractéristiques SocioEconomiques des Ménages Ruraux. MAEP : Cotonou, p. 84.

Mbetid-Bessane E. 2004. Apiculture, source de diversification des revenues des petits agriculteurs: cas du bassin cotonnier en Centrafrique. Tropicultura, 22(3): 156158. Disponible en ligne sur http://www.tropicultura.org/content/v22 n3.html.pdf

Mensah GA. 2008. La société d'abeilles. Cours d'apiculture multigraphié dispensé aux étudiants d'agronomie et de DESS faune. Université d'AbomeyCalavi : Abomey-Calavi. 
Molan P. 2001. Why honey is effective as a medicine. Bee World., 82(1): 22-40. Disponible en ligne sur http://www.citeseerx.ist.psu.edu/viewdo c/download

Olivier B. 2008. L'apiculture, outil de développement pour l'Afrique. In Miel Maya Magasine., 10: 5-8. Disponible en ligne sur http://www.maya.be/fr/.../28mayazine-10-1-apiculture-outil-dedeveloppement.aspx

Nombré I. 2003. Etudes des potentialités mellifères de deux zones du Burkina Faso : Garango (Province du Bouglou) et Nazinga (Province du Nahouri). Thèse de Doctorat, Université de Ouagadougou, Ouagadougou, p. 156.

Nombré I. 2011. L'apiculture au Burkina Faso. Communication, p. 8. Disponible en ligne sur http://www.apiservices.com/articles/fr/g uenange_nombre_issa.pdf

Paraïso A, Olodo GP, Tokoudagba S, Auteu R, Yegbemey RN, Sanni A. 2012a. Déterminants et contraintes de la production du miel dans le Nord-Ouest du Bénin: cas des communes de Natitingou et de Tanguiéta. Journal de la Recherche Scientifique de l'Université de Lomé, 14(1): 69-82. Disponible en ligne sur http://www.ajol.info/ index.php/jrsul/article/view/86759

Paraïso A, Sossou A, Iz-Haquou D, Yegbemey RN, Sanni A. 2012b. Perceptions and adaptations of beekeepers and honey hunters to climate change: the case of the communes of Natitingou and Tanguieta in Northwest of Benin. African Crop Science Journal, 20(2): 523-532. Disponible en ligne sur http://www.ajol.info/index.php/acsj/artic le/view/81676/71825.pdf

Paterson PD. 2008. L'Apiculture. Presses Agronomiques de Gembloux : Belgique.

Tamboura H, Kaboré H, Yaméogo SM. 1998. Ethnomédecine vétérinaire et pharmacopée Traditionnelle dans le plateau central du Burkina Faso : cas de la province du Passoré. Biotechnol. Agron. Soc. Environ., 2(3): 81-191. Disponible en ligne sur http://www.pressesagro.be/base/index.ph p/base/article/view/57/57.pdf

Tchoumboue J, Tchouamo IR, Pinta JY, Njia MN. 2001. Caractéristiques socioéconomiques et techniques de l'apiculture dans les hautes terres de l'ouest du Cameroun. Tropicultura, 19(3):141-146. Disponible en ligne sur http://www.tropicultura.org/content/v19 n3.html.pdf

Tsafack MAS, Muluh AG, Kamajou F, Verina I, Vabi BM. 2011. Etude comparative de la rentabilité de deux types d'apiculture au Nord-Ouest Cameroun. Tropicultura., 29(1): 3-7. Disponible en ligne sur http://www.tropicultura.org/text/v29n1/3 .pdf

Yédomonhan H, Akoègninou A. 2009. La production de miel à Manigri (Commnue de Bassila) au Bénin: enjeu et importance socio-économique. Int. J. Biol. Chem. Sci., 3(1): 125-134. http://dx.doi.org/10.4314/ijbcs.v3i1.4274 3

Yédomonhan H. 2009. Plantes mellifères et potentialités de production du miel en zones guinéenne et soudano-guinéenne au Bénin. Thèse de Doctorat, Université d'AbomeyCalavi, Abomey-Calavi, p. 294. 